\title{
The Influence of Particle Interactions on the Existence of Quantum Particles Properties
}

\author{
Tomer Shushi \\ Institute of Advanced Sciences, Haifa University, Haifa 27019, Israel
}

\begin{abstract}
We propose a novel model, based on two postulates, which provide new perspective on the fundamental forces using special and general relativity concepts. Many studies address the relations between the particles and the space time manifold, and the latter's physical structure, whether it is Continuous or Discrete. In the proposed model the properties of the particles are classical in the sense of general relativity, whereas their quantum properties are arises due to the experiments.
\end{abstract}

Key words: Interference pattern, particle's structure, quantum theory.

\section{Introduction}

Particles have both particle-like and wave-like properties, where quantum phenomenon arises due to their physical structure [1-3]. Under the following Postulates we obtain a novel structure of the quantum properties which reinterpret the origin of quantum phenomenon.

We consider the physical realm to be the $(3+1)$ space-time dimensions where any physical objects in the universe, in particular particles, are a curvature of the space time manifold. Furthermore, we assume (as a postulate) that any particle is a local $(3+1)$ field with vibration, and curvature, where without interactions it remains with the same properties.

\section{Model}

The model is constructed from the following two postulates:

(1) The particles are a function of the space time manifold, where all of the objects in the physical reality are of $(3+1)$ dimensions and not less.

(2) The discrete properties of a particle are arises only by interference between the particle and itself, other particles, or other fields.

Corresponding author: Tomer Shushi, Ph.D student, research fields: quantum theory, relativity theory and probability. E-mail: tomershushi@gmail.com.
Postulate 1 arises the importance of taking into account special relativity concepts (e.g. Lorentz-FitzGerald contraction, relativity of simultaneity, and time dilation) and general relativity concepts, for describing the particles. From this postulate we deduce that a free particle without any interaction should be describe with the form of Einstein field:

$$
G_{\mu v}=\kappa T_{\mu \nu}
$$

The significance of this structure is that quantum properties such as spin are not exists at all for a free particle. In the sequel we show that these properties arising due to interactions between the particles to themselves or other particles or fields, which Postulate two suggests. From above general structure of the quantum world let us define a tensor $A^{\alpha \beta}{ }_{\mu \nu}$ which represents the interaction (we refer it as an "imaginary force", which is a curvature of space time that is generated by at least two gravitational objects) between the particles, where in general:

$$
A_{\mu \nu}^{\alpha \beta} \neq A^{\alpha \beta}{ }_{\nu \mu} \text {. }
$$

By defining a particle $\psi$ with the equation $G_{\mu \nu}^{1}=\kappa T^{1}{ }_{\mu \nu}$ and a physical field $\phi$ with $G^{2}{ }_{\mu \nu}=\kappa T^{2}{ }_{\mu \nu}$ use simple algebraic calculation we have the following equations:

$$
\boldsymbol{\mathcal { G }}_{\mu \nu}^{12}=\boldsymbol{\tau}^{12}{ }_{\mu \nu} .
$$


where,

$$
\begin{aligned}
\boldsymbol{\mathcal { G }}^{12}{ }_{\mu \nu} & :=\mathrm{G}_{\mu \nu}{ }^{1}-\mathrm{A}_{\mu \nu} \mathrm{G}_{\mu \nu}{ }^{2} \\
\boldsymbol{\tau}^{12}{ }_{\mu \nu} & :=\kappa T_{\mu \nu}{ }^{1}-\mathrm{A}_{\mu \nu} \kappa T_{\mu \nu}{ }^{2} .
\end{aligned}
$$

Eq. (3) describes the interactions between the particle with the physical field $\phi$. It is clear that now $\boldsymbol{\tau}^{12}{ }_{\mu \nu}$, defined in Eq. (4), is not a stress energy tensor such as $T_{\mu \nu}$, thus we call $\boldsymbol{\tau}^{12}{ }_{\mu \nu}$ as a strength tensor. Now, for getting the spin property we recall it in general the interaction matrix $A_{\mu v}$, which is not symmetric, thus:

$$
\boldsymbol{\tau}^{12}{ }_{\mu \nu}-\boldsymbol{\tau}^{12}{ }_{\nu \mu}=\kappa T^{1}{ }_{\mu \nu}-A_{\mu \nu} \kappa T^{2}{ }_{\mu \nu}-\left(\kappa T^{1}{ }_{\nu \mu}-A_{\nu \mu} \kappa T^{2}{ }_{\nu \mu}\right)(5)
$$

recall that $T_{\mu \nu}^{1}$ and $T^{2}{ }_{\mu \nu}$ are symmetric tensors, so, in general $T_{\mu \nu}^{2}$ is not a vacuum solutions and general $A_{\mu v}$ we have:

$$
\boldsymbol{\tau}_{\mu \nu}^{12}-\boldsymbol{\tau}^{12}{ }_{\nu \mu}=\kappa T_{\mu \nu}^{2}\left(A_{\nu \mu}-A_{\mu \nu}\right) \neq 0
$$

which provides the spin tensor $\boldsymbol{S}^{\alpha \beta \mu}(x)$ from the following justification: From conservation laws we obtain:

$$
\partial_{v}\left(\boldsymbol{\tau}^{12}\right)^{\mu v}=0
$$

where, the four-momentum vector at time $t$ is $\boldsymbol{p}^{\mu}=\int d^{4} x \boldsymbol{T}^{\mu 0}(x, t)$, and it is well known that for getting, both, the orbital angular momentum and the spin angular momentum for a particle we need that the stress energy tensor would not be symmetric, i.e.,

$$
\begin{aligned}
& \partial_{\mu} \boldsymbol{S}^{\alpha \beta \mu}(\mathrm{x})=\left(\boldsymbol{\tau}^{12}\right)^{\beta \alpha}-\left(\boldsymbol{\tau}^{12}\right)^{\alpha \beta} \neq 0, \\
& \boldsymbol{\tau}^{12}{ }_{\mu \nu} \neq \boldsymbol{T}^{12}{ }_{\nu \mu} .
\end{aligned}
$$

where, $\boldsymbol{S}^{\alpha \beta \mu}(x)$ is the well known spin tensor, and $\boldsymbol{\tau}^{12}{ }_{\mu \nu}-\boldsymbol{\tau}^{12}{ }_{\nu \mu}$, gives the torque density showing the rate of conservation between the orbital angular momentum and spin. In the case that the stress energy is a symmetric tensor we get the orbital angular momentum and not the spin property.

Maxwell's equations could be arise from the model as follows: it was showed that the non-symmetric Riemannian tensor $\boldsymbol{R}_{\mu v}$ arose [4] due to the following relation of $\Gamma_{\alpha \beta}{ }^{\mu}$ field that defined an infinitesimal vector shifts $d A^{\mu}=-\Gamma_{\alpha \beta}^{\mu} A^{\alpha} d x^{\beta}$, then the Riemannian tensor was as follows:

$$
\boldsymbol{R}^{\alpha}{ }_{\mu . \nu \beta}=-\frac{\Gamma_{\mu \nu}^{\alpha}}{\partial x_{\beta}}+\Gamma_{\sigma \nu}^{\alpha} \Gamma_{\mu \beta}^{\sigma}+\frac{\Gamma_{\mu \beta}^{\alpha}}{\partial x_{v}}+\Gamma_{\mu \nu}^{\sigma} \Gamma_{\sigma \beta}^{\alpha}
$$

and hence the Ricci tensor takes the form:

$$
\begin{gathered}
\boldsymbol{R}_{\mu v}=\boldsymbol{R}^{\alpha}{ }_{\mu v \alpha}=- \\
\frac{\Gamma^{\alpha}{ }_{\mu v}}{\partial x_{\alpha}}+\Gamma^{\alpha}{ }_{\mu \beta} \Gamma^{\beta}{ }_{\alpha v}+\frac{\Gamma^{\alpha}{ }_{\mu \alpha}}{\partial x_{v}}+\Gamma^{\alpha}{ }_{\mu v} \Gamma^{\beta}{ }_{\alpha \beta}
\end{gathered}
$$

where, $\Gamma_{\mu v}^{\alpha}$ is in general not a symmetric tensor. From Einstein UFT this generality of the Riemannian tensor, $R^{\alpha}{ }_{\mu \nu}$, gives the Maxwell's equations, and hence

Maxwell's equations arise also in the proposed model since equation [5] can be formulated with the same $R_{\mu \nu}$, such as Einstein-Cartan theory [6, 7]. Moreover, if the metric of $g_{\mu \nu}$, [5] is skew-symmetric tensor the force which is describing is the electromagnetic force $[4,5,8]$.

\section{Structure of the Particles as Stationary in Space Time}

First, we consider the property of stationary for the space time manifold of the particle since we consider the particle to be a curvature and vibration of the space time manifolds, where this function of space time is stationary and does not depend on the time line, since these particles are supposed to remain with the same physical properties if they would be without any interactions.

Using the Newtonian limit and consider the metric $g_{\mu \nu}$ we get the well known equation for stationary space time:

$$
\phi=R
$$

where, $R$ is the Ricci scalar. Suppose that $R=((\mathrm{m} / \lambda))^{2} \varphi$, i.e., $\lambda$ is a parameter represents the curvature of the field, so

$$
\phi=\left(\frac{m}{\lambda}\right)^{2} \phi
$$

which takes the form of the Klein-Gordon equation 
which represents the particle's possible paths arises by the interaction between him to himself or other fields. Notice that according to our model $\varphi$ represents all of the particles, and not only a spin zero particle, since we consider the spin property of the particle to be a function of the GR properties of the particle and the properties of the physical environment of the experiments. The solutions of equation [7] provide discrete energy levels, which can be explained by interference pattern of the field himself or with other fields.

\section{References}

[1] Jensen, G. and To, S. 2013. Particle-Wave Duality and "Which-Way" Information.

[2] Lüth, H. 2013. "Particle-Wave Duality." Quantum Physics in the Nanoworld, 27-78. Springer Berlin Heidelberg.
[3] Elbaz, C. 2014. "Wave-Particle Duality in Einstein-de Broglie Programs." Journal of Modern Physics 5 (18): 2192.

[4] Einstein, A. 1925. "Einheitliche Feldtheorie von Gravitation Und Elektrizitat.”. Wiley-VCH Verlag GmbH \& Co. KGaA, 267-73.

[5] Ferraris, M. and Kijowski, J. 1982. Unified Geometric Theory of Electromagnetic and Gravitational Interactions. General Relativity and Gravitation, 14 (1): 37-47.

[6] Baekler, P., and Hehl, F. W. 2011. "Beyond Einstein-Cartan Gravity: Quadratic Torsion and Curvature Invariants with Even and Odd Parity Including All Boundary Terms." Classical and Quantum Gravity 28 (21): 215017.

[7] Trautman, A. 2006. "Einstein-Cartan Theory." arXiv preprint gr-qc/0606062.

[8] Sauer, T. 2006. "Field Equations in Teleparallel Space-Time: Einstein's Fernparallelismus Approach toward Unified Field Theory. Historia mathematica 33 (4): 399-439. 